\title{
Gene therapy for Rett syndrome: prospects and challenges
}

\author{
Kamal KE Gadalla ${ }^{*, 1,3}$, Paul D Ross', Ralph D Hector ${ }^{1}$, Noha G Bahey ${ }^{1,4}$, Mark \\ ES Bailey² \& Stuart R Cobb*,1
}

Rett syndrome (RTT) is a neurological disorder that affects females and is caused by lossof-function mutations in the X-linked gene MECP2. Deletion of Mecp2 in mice results in a constellation of neurological features that resemble those seen in RTT patients. Experiments in mice have demonstrated that restoration of MeCP2, even at adult stages, reverses several aspects of the RTT-like pathology suggesting that the disorder may be inherently treatable. This has provided an impetus to explore several therapeutic approaches targeting RTT at the level of the gene, including gene therapy, activation of $M E C P 2$ on the inactive $\mathrm{X}$ chromosome and read-through and repair of RTT-causing mutations. Here, we review these different strategies and the challenges of gene-based approaches in RTT.

Rett syndrome (RTT; MIM 312750) is a pediatric neurological disorder and is a predominant cause of severe mental retardation in girls, with an incidence of approximately 1 in 10,000 female births worldwide [1]. RTT is characterized by its almost exclusive occurrence in females, by onset of overt signs several months postnatally, and by a constellation of clinical diagnostic and associated features [1]. In the majority of cases RTT is caused by de novo germline mutations in the X-linked gene, MECP2 [2]. The structure and suspected functions of the encoded protein, $\mathrm{MeCP} 2$, have been reviewed recently [3,4]. A range of mouse models of RTT and MeCP2 dysfunction have been constructed [5-7] and in the Mecp2 knockout mouse the reversibility of the phenotype on restoration of Mecp2 gene function has been comprehensively demonstrated [8-10]. In light of this reversibility on intervention at the level of the gene, an obvious therapeutic avenue with translatable potential for use in RTT patients presented itself - gene therapy. Initial studies using this approach have highlighted both the potential for, and challenges involved in, its implementation [11,12]. The aim of this review is to discuss these challenges and to speculate on potential novel gene-based therapeutic options to treat $\mathrm{MeCP} 2$ disorders.

\section{The RTT clinical picture \& RTT-like features in Mecp2 mutant mice}

Diagnostic features of typical RTT include a highly characteristic developmental regression, with accompanying loss of hand skills, impaired mobility and speech and development of stereotypic hand movement automatisms [13]. Onset typically occurs several months postnatally, after a period of apparently normal early development. These diagnostic features distinguish RTT from the autism spectrum disorders, although there is some phenotypic overlap with the latter [14,15]. Associated features, such as microcephaly, respiratory/autonomic abnormalities, seizures, scoliosis, growth deficits and early hypotonia, are very prevalent in RTT.

'University of Glasgow, Institute of Neuroscience and Psychology, College of Medical, Veterinary and Life Sciences, Glasgow, UK

${ }^{2}$ School of Life Sciences, College of Medical, Veterinary \& Life Sciences, University of Glasgow, Glasgow, G12 8QQ, UK

${ }^{3}$ Pharmacology Department, Faculty of Medicine, Tanta University, Egypt

${ }^{4}$ Histology Department, Faculty of Medicine, Tanta University, Egypt

*Authors for correspondence: Tel.: 0141 3306490; Kamal.Gadalla@glasgow.ac.uk

\section{KEYWORDS:}

- adeno-associated virus

- gene therapy • MECP2

- Rett syndrome 
MECP2 is highly conserved between mammalian species with approximately $95 \%$ and approximately $89 \%$ identity in amino acid and nucleotide sequence, respectively, between human and mouse. Since most MECP2 mutations leading to RTT involve loss of function of the mutant allele, RTT can be modeled using Mecp 2 knockout (KO) mice. There are also now a number of Mecp2 knock-in (KI) mouse lines modeling specific human missense or nonsense mutations. Mecp2 mutant mouse models recapitulate many of the key features that characterize RTT in humans, although there are differences [5-9,16,17], reflecting both the phenotypic variability seen in patients and a somewhat lower severity in mice. Generally, hemizygous null (KO) males show overt signs, including motor impairment, tremor, breathing abnormalities and limb stereotypies, at about 5-6 weeks of age, and the severity of these signs progresses in an aggressive fashion until premature death at 10-20 weeks $[5,6,8]$. In contrast, heterozygous female mice display a RTT-like phenotype well into adult life (with onset at about 6 months of age), show slower symptom progression and have a near normal lifespan due to the mosaic expression of MeCP2 so that $50 \%$ of their cells express normal MeCP2 [5,6]. In these mouse models there is a moderate genotype-phenotype correlation - for example, models with MeCP2 protein-null phenotypes [5,6] are more severely affected than late-truncating [18] or knock-in missense $[16,17,19]$ models. While heterozygous female mice are the most appropriate genetic model for human RTT patients, their relative phenotypic severity is lower, and thus most studies to date have been carried out on male null mice, as their more severe phenotype early in life can be used to assess the efficacy of therapeutic interventions more readily and efficiently.

While RTT Mouse models have been extremely useful in studying the pathophysiology and molecular mechanisms of RTT they have their limitations: the knock-in mutation models tend not to be accurate models in terms of phenotype severity $[20,21]$ and the nature of $\mathrm{MeCP} 2$ and of the models renders rapid screening for drugs impacting on $\mathrm{MeCP} 2$ pathways impractical in mice. These limitations can be partially overcome by the use of human induced pluripotent stem cells (iPSCs) derived from RTT patients, which represent a valuable resource to study the detailed effects of different human mutations on cell functions and to study the neuronal phenotype associated with these mutations after iPS cell differentiation [22]. Differentiated neurons from RTT-derived iPSCs recapitulate several morphological features of RTT including reduced nuclear size and dendritic spine density [23-25], as well as several functional deficits, such as a decrease in the frequency and amplitude of spontaneous excitatory and inhibitory postsynaptic currents [23]. Neurons differentiated from RTT mouse iPSCs exhibit fewer action potentials, decreased action potential amplitude, diminished peak inward currents and higher input resistance [26]. In addition, astrocytes differentiated from human iPSCs provided the first evidence that human astrocytes are capable of expressing MeCP2 and that mutant astrocytes have noncell autonomous adverse effects on morphology and function of wild-type neurons [27], adding to growing evidence that glia may have an important role in RTT pathology [28]. However, although the use of iPSCs is promising in many respects, this cellular model of RTT has several limitations. First, $\mathrm{X}$ chromosome inactivation patterns (random vs nonrandom) tend to be inconsistent in RTT iPSC lines, which can influence the interpretation of phenotypic analysis of neurons differentiated from these lines [29,30]. In addition, using retroviral or lentiviral vectors for reprogramming iPSCs can potentially affect the expression of genes adjacent to integration sites [31]. Although most retroviral or lentiviral genes are silenced in iPSCs, there are incidences of re-expression of the viral genes in iPSCs or their derivatives that could be detrimental [32]. New strategies to reprogram cells in the absence of integrating viral vectors have been reported [33,34], which may obviate this problem. Finally, while iPSCs represent a robust platform for drug screening $[29,31]$ at a single cell level, any potential therapy will still ultimately require accurate in vivo modeling.

\section{Reversibility of the RTT phenotype \& treatment strategies}

Over the last 10 years, it has become clear that, at least in preclinical studies on mice, neurological and behavioral features resulting from abnormal early brain development are potentially reversible across a range of models of neurodevelopmental disorders [35-46]. The demonstrated reversibility of the Mecp 2 knockout phenotype in mice [8] has stimulated numerous groups to explore therapeutic approaches designed to reverse existing 
RTT-like dysfunction, to prevent or delay its onset or to ameliorate the phenotypic trajectory. Two main categories of intervention suggest themselves: targeting the primary underlying cause (i.e., a loss of function mutation in $M E C P 2)$ or targeting processes further downstream in the pathogenetic pathway. In the following sections we will focus mainly on attempts to tackle this disorder at the gene level.

\section{Gene therapy approaches}

For disorders resulting from loss of function mutations, the primary gene therapy approach is gene augmentation. In the case of RTT, this involves delivering a construct containing a wildtype (WT) copy of the $M E C P 2$ coding region to affected cells (i.e., predominantly brain neurons, here) in order to establish gene function in those cells (where previously it has been lacking), and in turn establishing improved function at the molecular, cellular, regional, network and wholeanimal level. Challenges to such approaches (Figure 1) include finding an appropriate way to control expression of the exogenously derived gene and ensuring high transduction efficiency in appropriate cells while minimizing transduction of inappropriate cells. Regulation of $\mathrm{MeCP} 2$ levels within transduced cells is a particularly thorny issue, as MeCP2 is dosage-sensitive and its overexpression in brain cells is known to cause a severe phenotype in both humans and mice [47-50]. In addition, gene therapy in females with X-linked dominant disorders has to accommodate the fact that, due to X chromosome inactivation (XCI), approximately $50 \%$ of transduced cells are already expressing normal levels of WT MeCP2.

\section{Gene therapy design - choice of vector \& regulatory elements}

Given that the RTT phenotype results mostly from effects on long-lived, postmitotic neurons of the central nervous system [5,9,51,52], it is clear that ex vivo strategies involving removal of cells from the patient and their subsequent replacement are not going to be viable. Hence, in vivo delivery of the therapeutic transgene to the brain is required. Vectors currently in use for gene therapy are largely based on retroviruses, especially lentivirus, and adeno-associated virus (AAV) [53,54].

Lentiviral vectors are able to transduce nondividing cells and drive stable long term expression in neurons [55]. However, these vectors cannot cross the blood-brain barrier (BBB), even after its disruption with mannitol [56], and have to be delivered by direct injection into the brain parenchyma. Additionally, they display very limited capacity to spread beyond the injection site [57]. There are also inevitable safety issues around their potential to cause insertional mutagenesis although nonintegrating versions have been developed [58]. Rastegar and colleagues demonstrated the potential for lentiviral transgene delivery to improve the phenotype of Mecp2-null neurons derived from neuronal stem cells in culture [59]. These lenti-treated neurons displayed more dendritic growth and branching than uninfected controls [59]. However, while lentiviral approaches have gone into clinical development for particular neurological disorders where discrete targeting of specific brain regions or nuclei may be appropriate [60], the ubiquitous nature of MeCP2 expression throughout the nervous system makes lentiviral technologies less appropriate in RTT unless the aim is to target a limited range of specific features of the disorder, for instance, focal targeting on brainstem nuclei to correct breathing problems [61,62].

AAV vectors have become an attractive vector of choice for targeting nervous system disorders [63]. AAV-based strategies for gene therapy have been developed for several disorders of both CNS and periphery [64-68], some of which are at an advanced preclinical stage or are in Phase 1/2 clinical trials, such as hemophilia B and Parkinson disease [69-72]. AAV vectors constructed with a serotype 9 capsid (AAV9 vectors) show a particular promise as they display high CNS transduction efficiency after systemic administration [73]. AAV9 is very promising in the case of RTT gene therapy as it can cross the blood-brain barrier, infect neurons efficiently and mediate long-term transgene expression [74-76]. Gadalla and colleagues showed that single stranded AAV9-mediated delivery of a WT copy of $M E C P 2$ driven by chicken $\beta$-actin promoter resulted in high brain transduction efficiency (Table 1) after direct brain injection into neonatal mice [11]. Transgene expression was mainly found in neurons and exogenously derived MeCP2 levels in these cells were at near physiological levels (approximately equal to endogenous MeCP2 levels). Moreover, the treated mice showed improved locomotion, amelioration of the trajectory of a RTT-like phenotype severity score and prolonged survival [11]. In the same study, systemic administration of 


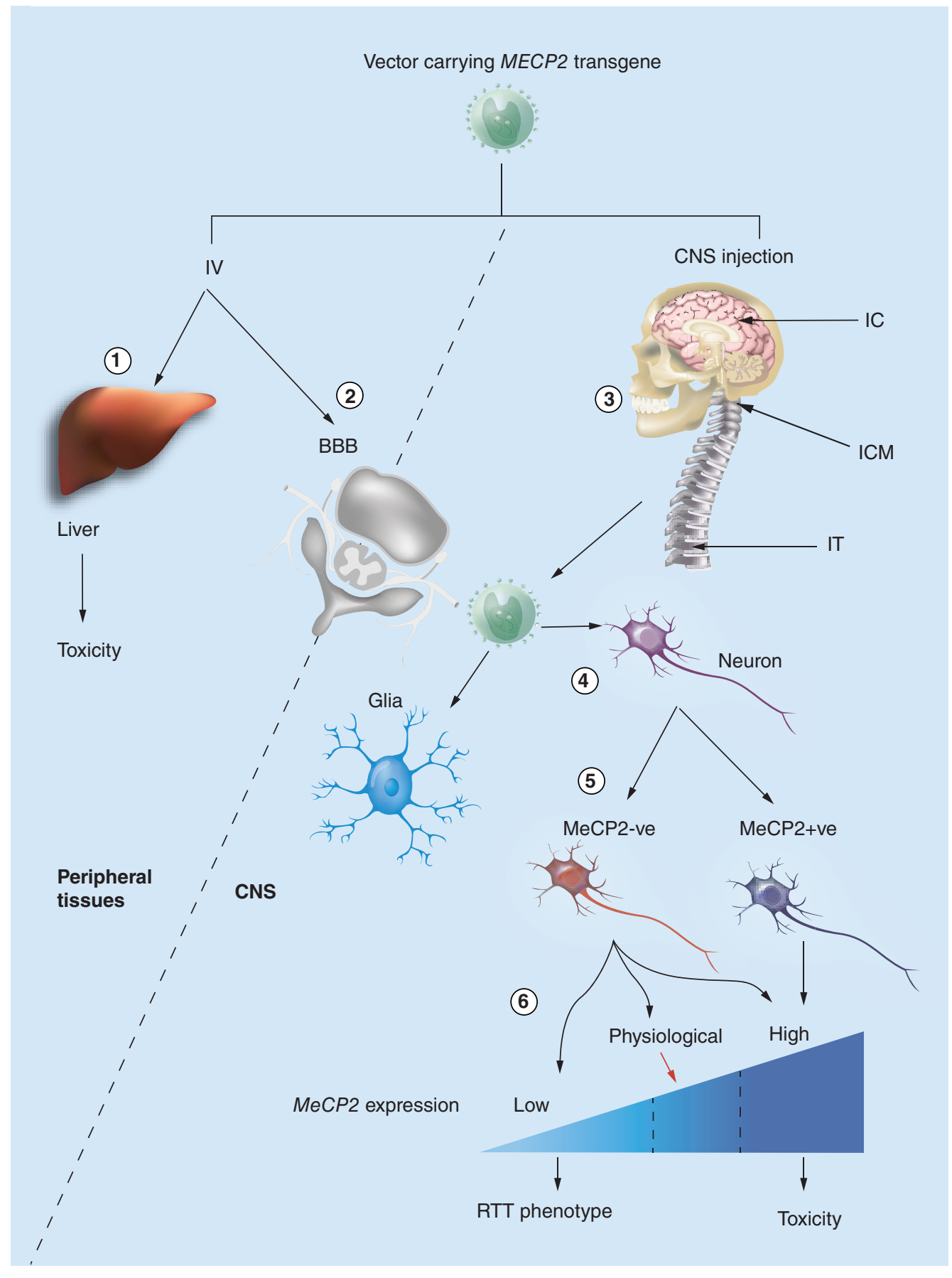

Figure 1. Summary of current challenges for RTT gene therapy. Intravenous (IV) injection of vector is likely to produce high peripheral expression, particularly in the liver (1), with potential accompanying toxicity. The BBB; 2) presents a challenge that prevents many types of vector from accessing the CNS. This problem can be overcome by direct CNS injection (3) using an IC route or routes that target the cerebrospinal fluid: ICM or IT injection. In the brain it is very important to transduce as many neurons as possible (4) to achieve maximal therapeutic effect. Among neurons, only neurons expressing the mutant allele and thus lacking functional MeCP2 should be targeted, ideally (5). In the transduced neurons the level of exogenous MeCP2 expression needs to be maintained at physiological levels (6) to avoid overexpression-related toxicity.

BBB: Blood-brain barrier; IC: Intracranial; ICM: Intra-cisterna magna; IT: Intrathecal. 
Table 1. Summary of experimental design and outcomes of published studies of gene therapy interventions in Rett syndrome mouse models.

\begin{tabular}{|c|c|c|c|c|}
\hline \multicolumn{5}{|l|}{ Gene therapy intervention } \\
\hline Age of treatment & Neonate & Adult [11] & Adult [12] & Adult [12] \\
\hline Sex & Male & Male & Male & Female \\
\hline Viruses & SsAAV9 & scAAV9 & scAAV9 & scAAV9 \\
\hline Promoter & CBA & MeP (229 bp) & MeP (730 bp) & MeP (730 bp) \\
\hline$M E C P 2$ isoform & Human MECP2_e1 & Human MECP2_e1 & Mouse Mecp2_e1 & Mouse Mecp2_el \\
\hline Route of administration & Intracranial & Intravenous & Intravenous & Intravenous \\
\hline Dose/mouse & $9.3 \times 10^{9} \mathrm{vg}$ & $5 \times 10^{11} \mathrm{vg}$ & $3 \times 10^{12} \mathrm{vg}$ & $3 \times 10^{12} \mathrm{vg}$ \\
\hline Transduction efficiency ( $\%$ of brain cells) & $\sim 40 \%$ & $\sim 2-4 \%$ of neurons & $\sim 25 \%$ & NA \\
\hline Results & $\begin{array}{l}\text { Prolonged survival } \\
\text { Reduced phenotype severity } \\
\text { Rescued nuclear volume } \\
\text { Improved locomotion }\end{array}$ & $\begin{array}{l}\text { Improved survival } \\
\text { Normalization of } \\
\text { GABA level }\end{array}$ & $\begin{array}{l}\text { Prolonged survival } \\
\text { Improved phenotype } \\
\text { Rescued soma size }\end{array}$ & $\begin{array}{l}\text { Stabilization of } \\
\text { phenotype }\end{array}$ \\
\hline
\end{tabular}

self-complementary AAV9-mediated delivery of a WT copy of MECP2 driven by a small (229 bp) fragment of the endogenous $M e c p 2$ promoter resulted in lower transduction efficiency in the brain, but was nevertheless associated with a survival advantage in male null mice. A second study reported similar findings for male mice and phenotype stabilization in heterozygous female mice [12].

For optimal efficacy and safety in gene therapy applications, there are two primary considerations - the proportion off target cells transduced, and the levels of transgene product in each transduced cell. In the case of RTT, genetic reversal experiments have demonstrated a strong correlation between amelioration of phenotype and the proportion of cells expressing MeCP2 after the reversal [10], while in the AAV9 studies [11,12], significant amelioration of the phenotype was apparent with transgene expression in only $25-40 \%$ of brain cells. However encouraging, this transduction efficiency was not able to completely rescue RTTlike phenotype to the wild-type level which probably requires higher transduction efficiency. As far as MeCP2 levels are concerned, it does appear that the cellular expression level is likely to be important. There is considerable evidence that reduced levels of functional MeCP2 $[17,77,78]$ lead to RTT-like phenotypes, while overexpression of $\mathrm{MeCP} 2$, as occurs in $M E C P 2$ duplication syndrome [47,48], also results in neurological effects $[19,49,50]$. The primary determinant of transgenic protein level is likely to be the promoter used in the construct. A study in which two promoters were compared, the well-characterized chicken $\beta$-actin promoter and the murine Mecp 2 endogenous core promoter [79], concluded that both are able to drive exogenous $\mathrm{MeCP} 2$ expression at near physiological levels.

Construct design, however, has received little attention thus far in relation to RTT gene therapy. Single-stranded AAV vectors have a payload capacity of approximately $4.7 \mathrm{~kb}$, while in scAAV vectors this is almost halved to $2.3 \mathrm{~kb}$ [80]. Given a MeCP2 coding region of $1.48 \mathrm{~kb}$, the space available for regulatory sequences at the $5^{\prime}$ - and $3^{\prime}$-ends is limited. Promoter fragments must be short if additional regulatory elements, which may help recapitulate endogenous expression patterns, are to be included [79,81]. Previous studies have shown differences in therapeutic outcomes when using different portions of the endogenous Mecp2 promoter constructs [11,12], suggesting that there is a crucial need to study Mecp2 regulatory elements in order to determine which elements are necessary to drive optimal expression of exogenous MeCP2.

$M E C P 2$ is regulated by a TATA-less promoter immediately upstream of exon 1 that lies within a CpG island. Systematic characterization of the mouse and human promoter regions, using deletion constructs and reporter assays, has identified potential core promoter regions, enhancer and silencer elements within a $1 \mathrm{~kb}$ region upstream of the coding region [81,82]. The MECP2 and $M e c p 2$ transcription start sites were previously predicted from RefSeq mRNAs and from 5'-RACE (Rapid Amplification of cDNA Ends) data [81], but have more recently been mapped from CAGE (Cap Analysis of Gene Expression) 
data as part of the FANTOM5 project [83]. These studies are in general agreement, all predicting transcription start sites approximately 60 nucleotides upstream of the exon 1 ATG start codon (used in the MECP2_e1 mRNA isoform), and thus defining a relatively small 5'-untranslated region (5'-UTR).

Other elements regulating MECP2 expression may lie further upstream, or downstream within or beyond the gene body. There have been analyses of potential regulatory elements in the flanking regions and potential regulatory elements have been identified from regions of high human and mouse sequence conservation [82] and by analysis of FANTOM5 CAGE data $[83,84]$. From these analyses, it is not apparent that there are strong neuronal MECP2specific enhancers, although some regulatory elements may be active in other cell types, and some potential elements may in fact be regulators of genes flanking $M E C P 2$. Further examination of these elements would be required to assess whether they ought to form part of an MECP2 gene therapy construct.

What might be the optimal time for gene therapy intervention?

RTT is thought to be a consequence of lack of neuronal maturation (to complete or to maintain maturation) and $\mathrm{MeCP} 2$ has been shown to play important roles in both neuronal development and maintenance processes [85-87]. In a mouse model, early restoration of $M e c p 2$ expression in presymptomatic mice led to apparent normal development [8]. Also, delayed activation of Mecp2 in adult symptomatic mice produced phenotypic recovery and normalization of the life span [8]. In another study, brain-specific expression of Mecp2 early in life showed prolonged lifespan, delayed phenotype development, improvement of motor activity and normalization of neuronal size [88], which are typically reduced in the Mecp2-null mouse [5]. From the available published preclinical gene therapy literature to date, it is clear that, delivery of MECP2 either in mouse neonates or in adult mice can produce survival and phenotypic benefits, indicating that therapeutic interventions can be effective across a wide range of ages. However, it is not yet clear whether there is a critical time window during which delivery of Mecp2 will produce the most robust improvement as this has so far not been tested in a systematic way. This may have important clinical considerations as early diagnostic testing improves. The appearance of the RTT phenotype several months postnatally (usually after 8-18 months) could present a particularly effective window for intervention before the onset of overt RTT features if early presymptomatic molecular diagnosis were to become practical and cost-effective. Such early treatment during infancy would also dramatically reduce the amount of viral vector required (if delivered peripherally) for treatment. However, the extent of amelioration of existing phenotypes after treatment of fully adult ( $\sim 10$ months) mice would predict that later administration to older girls or adults with RTT Syndrome would also be effective $[8,12,89]$. Regardless of the time of intervention, an important requirement is that transgene expression should be long-lasting, as deletion of Mecp2 from mature neurons is deleterious [87,90]. Indeed, there have been many reports of AAV-mediated transgenes persisting for long periods, including reports of continued expression $>6$ years after delivery in primates [91] and after $>8$ years in dogs $[92,93]$. Moreover, a recent gene therapy clinical trial has shown persistent gene expression for at least 10 years after AAVmediated gene delivery in human brain [94]

\section{Global or local delivery of MECP2}

Modeling RTT in mice has played a pivotal role in studying the requirement for $\mathrm{MeCP} 2$ in different brain areas $[9,88]$, cell types in the brain [95,96] and at different stages of development $[8,88]$, as well as in testing the 'reversibility' of RTT-like phenotypes [8]. The current consensus is that the brain is the key contributor to RTT pathology as brain-specific Mecp2 knockout leads to development of RTT-like phenotype and activation of endogenous Mecp 2 in the brain produces phenotype recovery $[8,9]$. It might be the case that lack of MeCP2 in the peripheral tissue produced an effect that is masked by the profound CNS-related phenotype. Indeed there is clinical and mouse data pointing to several phenotypes (bone, liver, lung) that may be peripheral in origin [97-101]. However, delivery of exogenous $M E C P 2$ directly to the brain using ssAAV9 [11], emphasizes the crucial role of the brain in ameliorating most of the gross aspects of the phenotype. Failure to correct some RTTlike features such as breathing abnormalities may reflect the insufficient transduction of cells in brain areas pertinent for those phenotypes (e.g., brainstem) or to lack of expression in the peripheral tissues that may contribute a given 
phenotype. Independent studies suggest that systemic delivery of exogenous MeCP2 (Table 1) using scAAV9 confers survival advantage despite low numbers of transduced cells in the brain. In the first report [11] $2-4 \%$ of neurons were transduced, with the majority of virus particles being taken up by peripheral tissues, particularly the liver. Increasing the dose by sixfold in another study [12] led to increased brain transduction ( $25 \%$ of cells) and greater phenotypic improvement in male null mice and phenotype stabilization in the heterozygous female mice. The potential significance of transgene expression within peripheral versus brain tissues remains to be established. These studies indicate the importance of achieving an appropriate therapeutic dosage. High viral titer is essential to get good coverage (a high proportion of cells infected) but, equally, cells being transduced by multiple viral particles could be detrimental because of the overexpression phenotype.

We have described the importance of restoring $\mathrm{MeCP} 2$ in the brain in order to achieve improvement of the RTT-like phenotype. Two methods can be used to deliver therapeutic constructs into the CNS: systemic delivery (e.g., intravenous injection) and resultant passage of the blood brain barrier, and direct CNS delivery routes (e.g., intrathecal, intracisterna magna or direct injection into the neuropil). Both approaches have been approved for clinical trials using AAV in other neurological disorders (https://clinicaltrials.gov $/$ ct $2 /$ results? term $=$ aav $\&$ Search $=\mathrm{Se}$ arch). Mecp2 reactivation experiments demonstrate a strong correlation between the percentage of cells in the brain expressing MeCP2 and the degree of phenotypic rescue [10]. Systemic administration of the vector, while perhaps more desirable from a translational standpoint, results in transduction of limited number of cells in the CNS and restricts the options for vector choice to those able to cross the BBB $[102,103]$. Moreover, any attempt to increase the dose to achieve more CNS expression will also be accompanied by increased levels of expression in peripheral tissues, particularly in the liver (Figure 1). Since many peripheral tissues normally express low levels of MeCP2 relative to neurons [52,104], this could lead to toxicity [11]. Modification of the vector construct to de-target the liver [105] and minimize the off-target effect and enhance CNS expression is crucial if systemic delivery is to be a feasible approach. Different strategies to achieve this are reviewed in detail in [106].
Considering the currently available vector technology, it will be very challenging to achieve high transduction efficiency (a large proportion of cells) in the brain after systemic administration. Therefore, direct CNS delivery is likely to be necessary to achieve appropriate CNS transduction. MeCP2 has a widespread action across the nervous system. This means that gene augmentation strategies have to target the whole brain instead of selectively targeting crucial brain regions. Trying to achieve widespread brain coverage while avoiding overexpression within the brain remains a formidable challenge and necessitates the development of appropriate promoter/regulatory elements in an attempt to gain some control over expression levels.

\section{Targeting specific cell types in the brain: neurons or astrocytes?}

$\mathrm{MeCP} 2$ is expressed in many tissues $[7,107,108]$ but is especially abundant in postmitotic neurons. The dominant neurological manifestation of the disorder indicates that neurons are more severely affected than other cell types [87]. However, there is growing recognition of the possible role of glial cells in the development of the phenotype [109,110] and its recovery [111]. It has been shown that mice lacking $\mathrm{MeCP} 2$ only in neurons show overt RTT-like symptoms while mice expressing $\mathrm{MeCP} 2$ only in neurons show an apparently normal phenotype [9]. This suggests that the bulk of the observable knockout phenotype comes from Mecp2 loss of function in neurons. This is also supported by the fact that phenotypic improvements after AAV9-mediated delivery of MECP2 in neonatal and adult mice was due mainly to expression in neurons [11,12].

As a further complication, several reports have shown that $\mathrm{MeCP} 2$ expression may be more important in certain neuronal subpopulations. For example, silencing $M e c p 2$ specifically in inhibitory GABAergic cells revealed a range of subtle RTT-like neuropsychiatric phenotypes including autistic-like repetitive behaviors [95]. In another study, in which Mecp2 was silenced in tyrosine hydroxylase-containing neurons, mice were found to display motor abnormalities and breathing problems including an increased incidence of apneas suggesting that dysfunction in aminergic systems may be responsible for some RTT-like breathing phenotypes [96]. Interestingly, preserved expression of $\mathrm{MeCP} 2$ 
with certain neuronal subtypes, in genetically engineered mice, showed extended survival and reduced phenotype severity [112-114]. Direct gene delivery to those cells using cell-specific promoters could be another option to treat specific aspects of the RTT-phenotype at the gene level.

\section{Exogenous MeCP2 level in transduced cells}

The ultimate goal of many gene therapy trials is to obtain high levels of transgene expression in order to achieve maximum therapeutic effect. However, this is not the case with RTT, where the level of exogenously derived $\mathrm{MeCP} 2$ within each cell needs to be maintained within physiological limits - this is one of the most challenging issues for RTT gene therapy (Figure 1). We know that cellular overexpression is a problem both from natural genetic experiments and from lab experiments on mouse and cellular models. In human patients, duplication of portions of $\mathrm{Xq} 28$ spanning the $M E C P 2$ locus is associated with a 'MECP2 duplication' phenotype characterized by neurological features including abnormal head growth, ataxia, seizures and mental retardation $[48,115,116]$. While mice with modest overexpression of transgenic MeCP2 show enhanced motor coordination, a reduced anxiety phenotype and increased context-dependent fear conditioning [49,50], mice expressing higher (two- to four-fold) levels of MeCP2 in neurons display tremors and motor dysfunction $[9,19]$. Gene therapy approaches will therefore have to deliver a therapeutic transgene to as many cells as possible while avoiding significant numbers of multiply-infected cells, in which overexpression would be a problem. In female patients the situation is even more challenging. Expression of exogenous $\mathrm{MeCP} 2$ in cells expressing the mutant allele is unlikely to cause problems, as recently reported [16], but expression in cells already expressing the WT allele is likely to result in overexpression in those cells.

Several strategies to circumvent such problems can be imagined. One strategy would be to use vectors that can specifically target cells expressing the mutant allele, perhaps by incorporating cell-specific ligands into the capsid or by finding other means of directing tropism toward MeCP2-deficient cells. Another strategy would be to engineer the vector construct so as to allow expression of the therapeutic transgene only in the absence of endogenous MeCP2 [3]. Interestingly, using AAV9-mediated delivery of MECP2 either in neonates or in adult mice showed no obvious overexpression-related toxicity [11,12]. Both studies showed levels of MeCP2 in transduced cells that were close to physiological, and the slightly raised levels of $\mathrm{MeCP} 2$ in treated wild-type mice, which express both endogenous and transgenic MeCP2, were well tolerated. While initial experiments in heterozygous female treated with AAV9/Mecp2 did not reveal overt problems of overexpression, further studies are required to address this important question.

\section{Other gene-targeted strategies in RTT}

Given the challenges confronting a gene therapy approach in RTT - the need to maintain and regulate $\mathrm{MeCP} 2$ expression levels in an approximately physiologically manner - an ideal therapy would avoid the need for provision of exogenously derived $\mathrm{MeCP} 2$, but would result in expression of endogenous WT MeCP2 under its normal endogenous regulatory control. One approach that can be used when RTT is caused by a nonsense mutation in the final exon is read-through pharmacotherapy. Here, mRNA produced from the mutant allele is translated under conditions where the premature stop codon is skipped, thus producing a protein that is WT at all residues except that encoded by the mutant codon. Several reports have shown that early-truncating ( $\mathrm{N}$-terminal to the boundary between the transcriptional repression domain and the C-terminal region) mutations, which account for approximately $40 \%$ of typical RTT patients [117], can be treated using gentamycin and related compounds that induce ribosomal read-through of premature stop codons [118,119]. In vitro experiments have demonstrated that full-length MeCP2 can be produced in cell lines harboring a variety of nonsense mutations after gentamycin treatment, although the efficiency of this process was generally rather low, ranging from 17 to $32 \%$ depending on the identity of the mutation and the effective concentration of gentamycin used $[120,121]$. The low efficiency of full-length $\mathrm{MeCP} 2$ production after aminoglycoside treatment, together with the well-known toxicity of the currently available aminoglycosides (nephrotoxicity and ototoxicity) indicates that this approach is not likely to be translational. In pursuit of such translationally beneficial approaches, other compounds with better safety profiles are therefore being tested instead. PTC12, NB54 and NB84 have all shown greater 
efficacy than gentamycin and with less toxicity [122-124]. However, little is known about the ability of these compounds to cross the BBB and so their likely efficiency in treating CNS disorders including RTT remains unknown.

Another approach would be to reactivate the $M E C P 2$ gene on the inactive $\mathrm{X}$ chromosome. As mentioned above, $M E C P 2$ is subject to $\mathrm{XCI}$, and each cell in a heterozygous female RTT patient therefore expresses either the WT allele or the mutant MECP2 allele, never both (Figure 2). This process is usually random and results in an approximately 50:50 mixture of cells of each type ('normal' and 'mutant'), although the XCI ratio varies between tissues and between individuals. In the brain, developmental processes ensure that normal and mutant cells are closely intermingled in a fine scale mosaic pattern with no tendency for clustering [8].

Whether a therapeutic strategy involving reactivation of the inactive $\mathrm{X}$ needs to be targeted only to the mutant cells depends on whether biallelic expression in a heterozygote results in dominant negative effects of the mutant allele or not. Two recent papers suggest that such effects do not occur, at least for some mutations [16,21]. Thus, the strategy might simply involve reactivating the inactive $\mathrm{X}$ in all cells. Activation of X-linked genes has been shown to be possible by reduction of genomic methylation levels using 5-azacytidine in cell culture systems [125], although this type of blunt instrument approach is not thought likely to be of therapeutic utility. Recently, a study using small molecules to inhibit $\mathrm{X}$-chromosome inactivation factors showed $\mathrm{MeCP} 2$ expression resulting from the inactive $\mathrm{X}$ harboring the normal allele in cultured fibroblasts taken from RTT patients [126]. This pharmacological activation of the inactive $\mathrm{X}$ is reversible and therefore the reactivation requires the continued presence of the drug. For inactive $\mathrm{X}$ reactivation to work as a therapeutic strategy in RTT, several important hurdles have to be surmounted. First, in cells that currently express the normal allele, reactivation of the silent $\mathrm{X}$ will lead to subsequent and potentially detrimental expression of the mutant allele. However, recent evidence suggests that there are unlikely to be disruptive interactions between wild-type and mutant domains of $\mathrm{MeCP} 2$ when co-expressed in the same cells (Figure 2) [16]. Second, the safety and CNS bioavailability properties of potential small molecule X-chromosome activators would need to be within acceptable limits. Third, the efficiency and the proportion of cells expressing normal MeCP2 from the inactive X chromosome, as well as the dose dependency of expression, is currently unknown and will need to be assessed in detail. Finally, a major problem with this approach is that if the entire inactive $\mathrm{X}$ is reactivated, the $\mathrm{X}$-linked gene dosage problem, for which X inactivation has presumably evolved, will appear and pathological levels of gene expression are likely to result. However, female mice lacking STC1 (an X chromosome inactivation factor) express genes from both $\mathrm{X}$ chromosomes, but at normal levels with apparently normal phenotype suggesting the presence of previously unknown compensatory mechanisms [126]. It is not known whether similar compensatory measures will occur in humans. However, the fact that the process of pharmacologically-induced X-chromosome reactivation is reversible does have some safety benefits compared with other gene-based interventions.

Each of the possible solutions discussed above is associated with the challenge presented by the continued presence of the mutant allele in the cell types affected. The most conceptually attractive, but potentially technically challenging gene-based approach would be to edit the mutation at the genomic level and turn it into a WT allele, which would then perform its normal function for the remainder of the life of that cell, and potentially therefore the lifetime of the patient. Such genome editing approaches were inconceivable until very recently, but the explosion of work in this area of the last couple of years has opened the door to development of such approaches. Several editing agents are currently being developed, including TALENs and the CRISPR/Cas9 system. The latter has emerged as the genome editing system of choice due to its efficiency, ease of synthesis and ability to target multiple loci simultaneously $[127,128]$. In bacteria, the CRISPR system along with its associated CRISPR-associated (Cas) nuclease proteins provides a form of adaptive immunity by targeting nucleases against invading viral DNA [129,130]. The Cas nuclease is targeted to specific sequences in the invading nucleic acid via RNA guides. A combination of Cas nuclease (usually Cas9) and target-specific RNA guide can be used to target double-stranded or staggered DNA breaks to a huge range of sites within any mammalian genome. Once a DNA break has occurred, the normal DNA repair pathways in the cell can then be hijacked to produce 


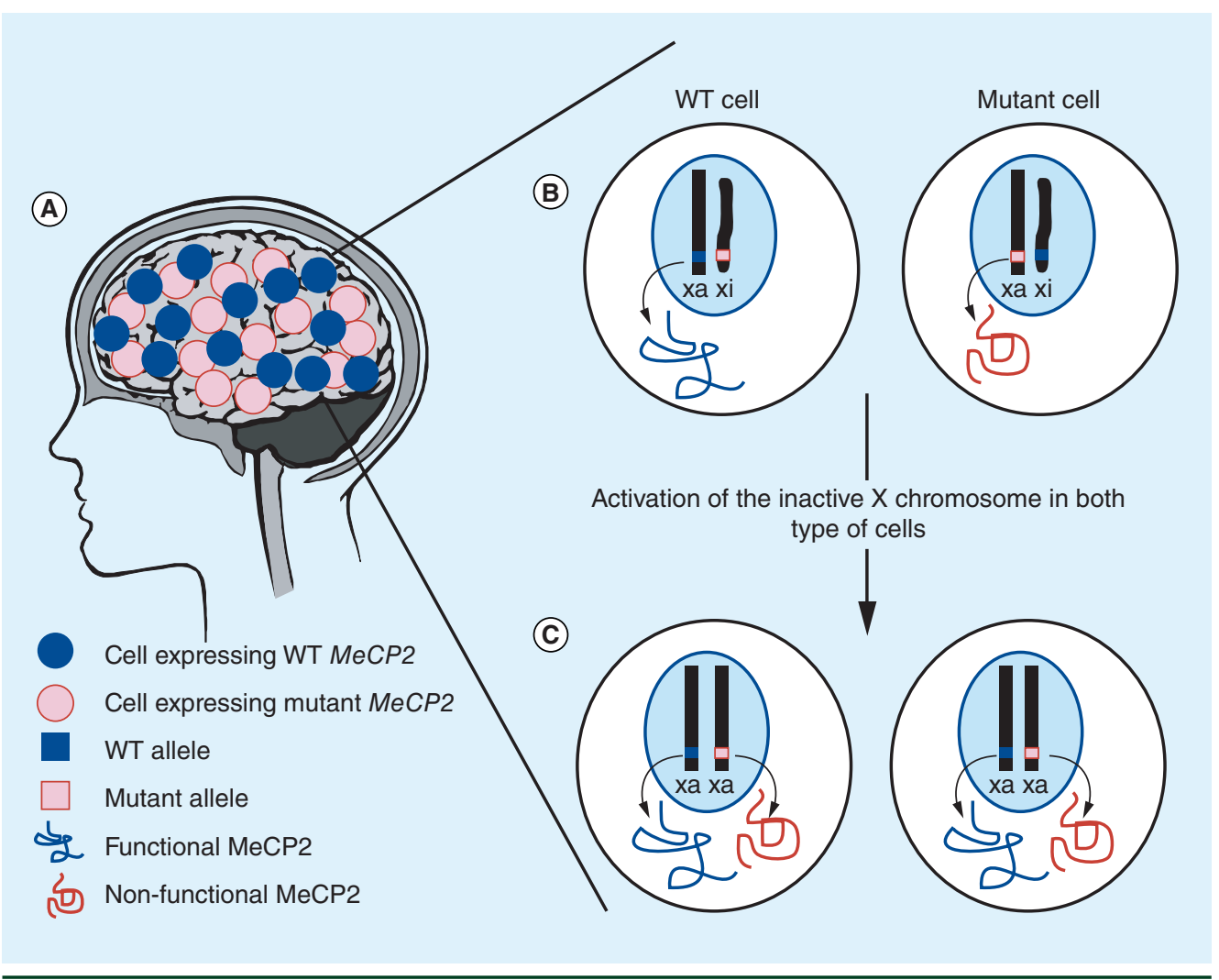

Figure 2. Activation of the inactive $\mathrm{X}$ chromosome. (A) Diagram of the brain of a female Rett syndrome patient with mosaic expression of MeCP2 due to random X chromosome inactivation. (B) If the WT allele is located on the active $\mathrm{X}$ chromosome the cell will express functional MeCP2, whereas a cell with the mutant allele located on the active $X$ chromosome will express nonfunctional MeCP2. (C) Activation of the inactive X chromosome in all cells will lead to expression of both WT and mutant alleles, thus leading to cells containing both WT and mutant MeCP2 protein WT: Wild-type; xa: active X chromosome; xi: inactive X chromosome.

desired genetic changes, either precise changes utilizing the homologous recombination (HR) pathway or mutagenic deletions and transgene insertions using end-joining (EJ) pathways of various types. While this technology has been mostly harnessed for the quick and easy generation of new animal models [131] and for probing gene function [132] its potential as a therapeutic agent is clear.

Many of the disease-causing mutations in RTT are single base changes. An attractive repair strategy would be to precisely change the mutated base to the WT version by using the HR repair pathway and an exogenously-delivered homologous repair template (Figure 3 ). This strategy has been used successfully to repair the disease-causing mutation in cultured cells from cystic fibrosis patients [133] and in adult mouse models of human hereditary tyrosinemia, leading to partial rescue of the phenotype [134].
Crucially, however, in such studies either an ex vivo approach is used where successfully edited cells can be expanded in culture before returning them to the body, or the edited cells are able to divide and also have a selective advantage. In contrast, in RTT the major target cells are postmitotic neurons in the brain and therefore this selection process is not available $[5,6]$. In addition, nondividing cells such as neurons have very low levels of HR and rely mainly on the nonhomologous end joining (NHEJ) repair pathway $[135,136]$ meaning that the vast majority of DNA breaks induced by the CRISPR/Cas9 system will lead to further mutagenic changes due to the error-prone nature of this pathway. In addition, CRISPR RNA guides can tolerate several mismatches in binding to their target $[137,138]$ and this approach therefore cannot easily be made mutant allele-specific or free from off-target effects at other loci. In addition, the 
small insertions and deletions created by NHEJ repair are likely to destroy the CRISPR target site thus preventing any chance of later repair in subsequent treatment cycles.

Some studies have suggested that non-neuronal CNS cells such as astrocytes and microglia play a crucial role in the RTT phenotype and that repair of these cells alone provides robust phenotype amelioration [111,139,140]. While HR levels in these dividing cells will be higher than in neurons it will not be enough to compensate for the vastly greater number of cells that will be repaired imprecisely by NHEJ. In a recent study employing a high-throughput screen to identify chemicals capable of modulating HR-mediated genome editing, a small molecule (L-755507) was identified that could enhance HR repair by up to ninefold [141]. However even with this increase, HR repair levels only reached a peak of $6 \%$ compared with up to $57 \%$ of NHEJ-mediated mutagenic events. As more becomes known about how these competing pathways are regulated, it may be possible to selectively enhance $\mathrm{HR}$, at the expense of NHEJ, for a brief window to allow HR-mediated repair to occur. Currently, however, precise editing of point mutations does not

(A)

Safe genomic location

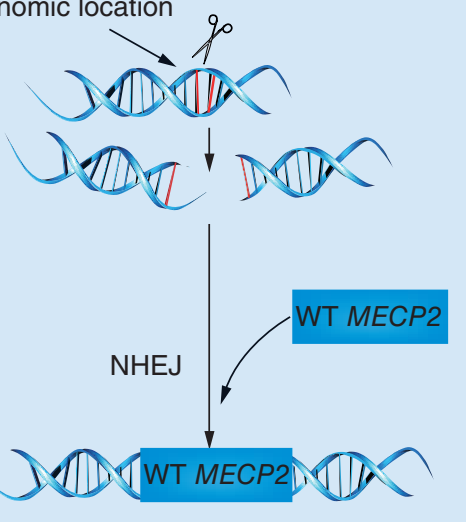

appear to be a viable therapeutic route for RTT. An alternative approach is to utilize the fact that NHEJ predominates in neurons and develop methods that employ this repair system. In one recent study, Maresca and colleagues [142] demonstrated that large DNA sequences can be captured at nuclease-induced double stranded breaks if this DNA is flanked by the same targeting sequences as the chromosomal DNA. This process was found to be NHEJ-dependent and provides a robust way to ligate DNA constructs into precise locations in the genome. One therapeutic strategy would be to use this process to ligate a WT copy of the MECP2 coding sequence into the AAVS1 safe harbor locus, a site in the human genome known to allow stable transgene expression [143]. Similar to current gene therapy approaches, this strategy enables the cell to express functional protein without the risk of damaging WT copies of the gene in nonmutant cells. However, an advantage of this approach over current gene therapy strategies employing AAV vectors is that the transgene will be permanently integrated into the genome, allowing dividing cells such as astrocytes and microglia, and perhaps neuronal stem cells also, to pass the WT transgene on to

\section{(B)}

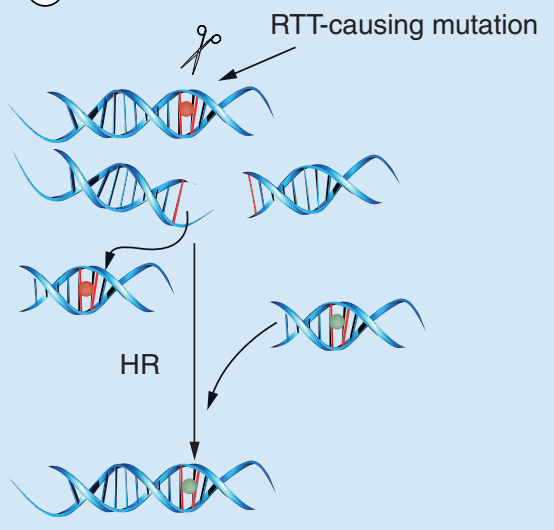

Figure 3. Mechanism of action of CRISPR/Cas system. In the CRISPR/Cas9 genome editing system a guide RNA (gRNA) binds to a target sequence and creates a template for the Cas9 nuclease to make a targeted, double-stranded break in the DNA, leading to activation of one of the two major repair pathways that can be exploited for therapy. (A) When the NHEJ pathway is activated, DNA ends are ligated together without the need for a homologous DNA template. This pathway could be used to ligate a WT copy of the MECP2 gene into a safe harbor in the genome. (B) In the presence of the HR machinery in dividing cells, homology-directed repair occurs and an exogenously provided WT template can be inserted accurately into the genome in place of a region containing a disease-causing mutation, thus editing the mutant allele (red) and causing WT MeCP2 to be expressed instead (green). HR: Homologous recombination; NHEJ: Nonhomologous end joining; RTT: Rett syndrome; WT: Wildtype. 


\section{EXECUTIVE SUMMARY}

- Rett syndrome (RTT) presents a major cause of mental retardation and motor disabilities in females.

- $\quad$ RTT is mainly caused due to loss of functional mutation of X-linked gene MECP2.

Reversibility of the RTT phenotype \& treatment strategies

- Delayed activation of endogenous Mecp2 in mouse model resulted in phenotype recovery.

Gene therapy design - choice of vector \& regulatory elements

- AAV9-mediated delivery of MECP2 showed ability to transduce brain cells in Mecp2 knock out mice and showed therapeutic benefits.

- Using ubiquitous or Mecp2 promoter fragments in two different studies produced near normal physiological level of MeCP2 with no apparent overexpression-related toxicity.

What might be the optimal time for gene therapy intervention?

- AAV9-mediated delivery of MECP2 showed survival advantages and phenotype improvement when applied into neonatal or adult mice.

- Gene therapy interventions can be effective across a wide range of ages.

\section{Global or local delivery of $M E C P 2$}

- Brain is the main contributor to RTT phenotype and treatment.

- Direct brain injection of AAV9/MECP2 in neonatal mice showed high transduction efficiency compared with systemic delivery in adult mice.

- Systemic administration will be accompanied by high transduction in the peripheral organs which could be toxic.

Target specific cell type in the brain; neurons or astrocytes

- Within the brain, neurons play a key role in RTT development and rescue.

- Certain neuronal subpopulation might have more roles in the development of RTT.

Exogenous MeCP2 level in the transduced cells

- MECP2 duplication in human and mouse model results in many neurological deficits.

- No apparent toxicity was reported after AAV9-mediated delivery of MECP2.

- Targeting exogenous MECP2 to cells expressing the mutant allele is crucial to avoid overexpression-related toxicity when treating heterozygous females.

Other gene-targeted strategies in RTT

- Mecp2 early truncation mutation can be pharmacologically overcome to produce full length protein in vitro.

- Activation of the inactive $X$ chromosome in culture cells showed expression of normal MeCP2 in cells expressing the mutant allele.

- Overdosage issues with other X-linked genes and in vivo efficiency are still to be investigated before being considered for translational applications.

- Recently development of CRISPR/Cas9 technology might be applicable to correct MECP2 mutations.

Conclusion

- Neurological manifestations of RTT are correctable at the gene level in mouse models.

- CNS, particularly neurons, is the main player in RTT pathology and recovery.

- AAV9-mediated delivery of MECP2 in mouse models showed phenotype improvement and survival advantages.

- Cell-specific targeting of MECP2 and maintaining exogenous MeCP2 expression close to the physiological level in as many cells as possible are the current key challenges in the field of RTT gene therapy. 
daughter cells. Crucially, the targeted nature of the insertion helps to avoid the problem of insertional mutagenesis, which is a major concern for other insertional approaches such as delivery using lentiviral vectors.

Delivering CRISPR editing tools selectively to the nervous system presents as big a challenge as it does in traditional gene therapy. The Cas 9 coding region is too big to be packaged effectively into an AAV vector alongside the required guide RNA and necessary promoters and termination sequences [132], necessitating delivery of the reagents in multiple vectors. This will have a significant impact on the number of cells that can realistically be targeted, as multiple transduction events will be needed in each cell. However, the currently used CRISPR/Cas9 system is only one of an array of CRISPR type systems employed by different bacteria [144]. Indeed, very recently Ran and colleagues carried out a screen to identify possible alternatives to the Cas9 from Streptococcus pyogenes [145]. Excitingly, they found a Cas9 protein from Staphylococcus aureus that appears to have similar efficiencies to the $S$. pyogenes version. Crucially, it is only $3.2 \mathrm{~kb}$ long, which allowed it to be deployed along with an RNA expression cassette in a single vector approach using ssAAV. If replicated, this finding could greatly enhance our ability to target cells of the nervous system efficiently with CRISPR tools. Genome editing technologies hold great promise in tackling the scourge of genetic disease and the astonishing pace of development in the past few years suggests that these tools will have much to offer us in the future. However, many challenges have to be overcome before it becomes a viable treatment option for a complex brain disorder like RTT.

\section{Conclusion}

Substantial progress has been made in understanding the molecular basis of RTT and proofof-concept studies have shown that phenotype improvement is possible by delivering an $M E C P 2$ transgene. Significant progress is being made on a number of fronts; attempts to restore endogenous Mecp2 expression, reactivation of the inactive $\mathrm{X}$ chromosome and pharmacological approaches to 'read-through' truncation mutations have all shown some progress in vitro. However, there is, as yet, no strong evidence that these approaches can work efficiently in vivo and thus gene augmentation using viral vectors remains the most likely approach to facilitate translation to clinical development in the medium term, if vector optimization for efficacy and safety can be achieved. Particular efforts need to be directed to the development of vectors with greater CNS transduction efficiency and larger payload capacity. The opportunities and challenges in making gene-based approaches a reality in RTT are energizing an optimistic research effort toward this end.

\section{Future prospects}

Studies to date have provided solid evidence that gene-based approaches have significant potential to impact upon key features of RTT. The major challenges ahead include the development of vectors that can efficiently deliver a transgene or gene correction reagents to large number of cells in the brain as well as constructs that will ensure acceptable levels of $\mathrm{MeCP} 2$ expression.

\section{Financial \& competing interests disclosure}

We are grateful to the Rett Syndrome Research Trust, Reverse Rett, Scottish Government Chief Scientist Office and the Rett Syndrome Association Scotland for support. The authors have no other relevant affiliations or financial involvement with any organization or entity with a financial interest in or financial conflict with the subject matter or materials discussed in the manuscript apart from those disclosed.

No writing assistance was utilized in the production of this manuscript.

\section{References}

Papers of special note have been highlighted as:

- of interest; $\bullet$ of considerable interest

1 Neul JL, Kaufmann WE, Glaze DG et al. Rett syndrome: revised diagnostic criteria and nomenclature. Ann. Neurol. 68(6), 944-950 (2010).

- Revision of the diagnostic criteria for Rett syndrome (RTT) and review of MECP2 mutations as a cause of typical and atypical RTT.

2 Amir RE, Van Den Veyver IB, Wan M, Tran CQ, Francke U, Zoghbi HY. Rett syndrome is caused by mutations in $\mathrm{x}$-linked mecp2, encoding methyl-cpg-binding protein 2. Nat. Genet. 23(2), 185-188 (1999).

3 Gadalla KK, Bailey ME, Cobb SR. Mecp2 and Rett syndrome: reversibility and potential avenues for therapy. Biochem. J. 439(1), 1-14 (2011).

4 Lyst MJ, Bird A. Rett syndrome: a complex disorder with simple roots. Nat. Rev. Genet. 16(5), 261-275 (2015).

5 Chen RZ, Akbarian S, Tudor M, Jaenisch R. Deficiency of methyl-cpg binding protein-2 in cns neurons results in a rett-like phenotype in mice. Nat. Genet. 27(3), 327-331 (2001). 
-• Creation of mouse model of RTT syndrome and characterization of the RTT-like phenotype in this model.

6 Guy J, Hendrich B, Holmes M, Martin JE, Bird A. A mouse mecp2-null mutation causes neurological symptoms that mimic Rett syndrome. Nat. Genet. 27(3), 322-326 (2001).

7 Shahbazian M, Young J, Yuva-Paylor L et al. Mice with truncated mecp2 recapitulate many rett syndrome features and display hyperacetylation of histone h3. Neuron 35(2), 243-254 (2002).

-• Reversibility of RTT-like phenotype after delayed activation of endogenous $M e c p 2$ globally in mouse model of RTT.

8 Guy J, Gan J, Selfridge J, Cobb S, Bird A. Reversal of neurological defects in a mouse model of rett syndrome. Science 315(5815), 1143-1147 (2007).

- Importance of MeCP2 expression in neurons and demonstrated reversibility after expression of MeCP2 in the neurons only.

9 Luikenhuis S, Giacometti E, Beard CF, Jaenisch R. Expression of mecp2 in postmitotic neurons rescues rett syndrome in mice. Proc. Natl Acad. Sci. USA 101(16), 6033-6038 (2004)

10 Robinson L, Guy J, Mckay L et al. Morphological and functional reversal of phenotypes in a mouse model of rett syndrome. Brain 135(Pt 9), 2699-2710 (2012).

- Phenotype improvement and survival advantages in $M e c p 2$ knockout mice after AAV9 mediated delivery of MECP2.

11 Gadalla KK, Bailey ME, Spike RC et al. Improved survival and reduced phenotypic severity following aav $9 /$ mecp 2 gene transfer to neonatal and juvenile male mecp2 knockout mice. Mol. Ther. 21(1), 18-30 (2013).

-• Rescue of adult male knockout and heterozygous female mice after systemic delivery of AAV9/Mecp2.

12 Garg SK, Lioy DT, Cheval H et al. Systemic delivery of mecp2 rescues behavioral and cellular deficits in female mouse models of rett syndrome. J. Neurosci. 33(34), 1361213620 (2013)

13 Neul JL, Kaufmann WE, Glaze DG et al. Rett syndrome: revised diagnostic criteria and nomenclature. Ann. Neurol. 68(6), 944-950 (2010).

14 Jedele KB. The overlapping spectrum of rett and angelman syndromes: a clinical review. Semin. Pedriatr. Neurol. 14(3), 108-117 (2007).
15 Castro J, Mellios N, Sur M. Mechanisms and therapeutic challenges in autism spectrum disorders: insights from Rett syndrome. Curr. Opin. Neurol. 26(2), 154-159 (2013).

16 Heckman LD, Chahrour MH, Zoghbi HY. Rett-causing mutations reveal two domains critical for mecp2 function and for toxicity in mecp2 duplication syndrome mice. eLife 3 , doi:10.7554/eLife.02676 (2014).

17 Goffin D, Allen M, Zhang L et al. Rett syndrome mutation mecp $2 \mathrm{t} 158 \mathrm{a}$ disrupts DNA binding, protein stability and erp responses. Nat. Neurosci. 15(2), 274-283 (2012).

18 Moretti P, Levenson JM, Battaglia F et al. Learning and memory and synaptic plasticity are impaired in a mouse model of Rett syndrome. J. Neurosci. 26(1), 319-327 (2006).

19 Chao HT, Zoghbi HY. Mecp2: only 100\% will do. Nat. Neurosci. 15(2), 176-177 (2012).

20 Goffin D, Zhou ZJ. The neural circuit basis of Rett syndrome. Front. Biol. (Beijing) 7(5), 428-435 (2012).

21 Pitcher MR, Herrera JA, Buffington SA et al. Rett syndrome like phenotypes in the $\mathrm{r} 255 \mathrm{x}$ mecp2 mutant mouse are rescued by mecp 2 transgene. Hum. Mol. Genet. 24(9), 2662-2672 (2015).

22 Hotta A, Cheung AY, Farra N et al. Eos lentiviral vector selection system for human induced pluripotent stem cells. Nat. Protoc. 4(12), 1828-1844 (2009).

23 Marchetto MC, Winner B, Gage FH. Pluripotent stem cells in neurodegenerative and neurodevelopmental diseases. Hum. Mol. Genet. 19(R1), R71-76 (2010).

24 Ananiev G, Williams EC, Li H, Chang Q. Isogenic pairs of wild type and mutant induced pluripotent stem cell (ipsc) lines from Rett syndrome patients as in vitro disease model. PLoS ONE 6(9), e25255 (2011).

25 Kim K, Zhao R, Doi A et al. Donor cell type can influence the epigenome and differentiation potential of human induced pluripotent stem cells. Nat. Biotechnol. 29(12), 1117-1119 (2011).

26 Farra N, Zhang WB, Pasceri P, Eubanks JH, Salter MW, Ellis J. Rett syndrome induced pluripotent stem cell-derived neurons reveal novel neurophysiological alterations. Mol. Psychiatry 17(12), 1261-1271 (2012).

27 Williams EC, Zhong X, Mohamed A et al. Mutant astrocytes differentiated from rett syndrome patients-specific ipscs have adverse effects on wild-type neurons. Hum. Mol. Genet. 23(11), 2968-2980 (2014).
28 Andoh-Noda T, Akamatsu W, Miyake K et al. Differentiation of multipotent neural stem cells derived from rett syndrome patients is biased toward the astrocytic lineage. Mol. Brain 8, 31 (2015).

29 Marchetto MC, Carromeu C, Acab A et al. A model for neural development and treatment of rett syndrome using human induced pluripotent stem cells. Cell 143(4), 527-539 (2010).

30 Cheung AY, Horvath LM, Grafodatskaya D et al. Isolation of mecp2-null Rett syndrome patient hips cells and isogenic controls through $\mathrm{x}$-chromosome inactivation. Hum Mol. Genet. 20(11), 2103-2115 (2011).

31 Dajani R, Koo SE, Sullivan GJ, Park IH Investigation of rett syndrome using pluripotent stem cells. J. Cell. Biochem. 114(11), 2446-2453 (2013).

32 Okita K, Ichisaka T, Yamanaka S. Generation of germline-competent induced pluripotent stem cells. Nature 448(7151), 313-317 (2007).

33 Feng B, Ng JH, Heng JC, Ng HH. Molecules that promote or enhance reprogramming of somatic cells to induced pluripotent stem cells. Cell Stem Cell 4(4), 301-312 (2009).

34 Page RB, Voss SR. Induction of metamorphosis in axolotls (ambystoma mexicanum). Cold Spring Harb. Protoc. 2009(8), pdb prot5268 (2009).

35 Costa RM, Federov NB, Kogan JH et al. Mechanism for the learning deficits in a mouse model of neurofibromatosis type 1 . Nature 415(6871), 526-530 (2002).

36 Bourtchouladze R, Lidge R, Catapano R et al. A mouse model of rubinstein-taybi syndrome: defective long-term memory is ameliorated by inhibitors of phosphodiesterase 4. Proc. Natl Acad. Sci. USA 100(18), 10518-10522 (2003)

37 Alarcon JM, Malleret G, Touzani K et al. Chromatin acetylation, memory, and ltp are impaired in cbp $+/$ - mice: a model for the cognitive deficit in rubinstein-taybi syndrome and its amelioration. Neuron 42 (6), 947-959 (2004)

38 Li W, Cui Y, Kushner SA et al. The hmg-coa reductase inhibitor lovastatin reverses the learning and attention deficits in a mouse model of neurofibromatosis type 1. Curr. Biol. 15(21), 1961-1967 (2005).

39 Mcbride SM, Choi CH, Wang Y et al. Pharmacological rescue of synaptic plasticity, courtship behavior, and mushroom body defects in a drosophila model of fragile x syndrome. Neuron 45(5), 753-764 (2005). 
40 Dolen G, Osterweil E, Rao BS et al. Correction of fragile $\mathrm{x}$ syndrome in mice. Neuron 56(6), 955-962 (2007).

41 Fernandez F, Morishita W, Zuniga E et al. Pharmacotherapy for cognitive impairment in a mouse model of down syndrome. Nat. Neurosci. 10(4), 411-413 (2007).

42 Van Woerden GM, Harris KD, Hojjati MR et al. Rescue of neurological deficits in a mouse model for angelman syndrome by reduction of alphacamkii inhibitory phosphorylation. Nat. Neurosci. 10 (3), 280-282 (2007).

43 Ehninger D, Han S, Shilyansky C et al. Reversal of learning deficits in a tsc $2+/$ mouse model of tuberous sclerosis. Nat. Med. 14(8), 843-848 (2008).

44 Meikle L, Pollizzi K, Egnor A et al. Response of a neuronal model of tuberous sclerosis to mammalian target of rapamycin (mtor) inhibitors: effects on mtorcl and akt signaling lead to improved survival and function. $J$. Neurosci. 28(21), 5422-5432 (2008).

45 Rueda N, Florez J, Martinez-Cue C. Chronic pentylenetetrazole but not donepezil treatment rescues spatial cognition in ts $65 \mathrm{dn}$ mice, a model for down syndrome. Neurosci. Lett. 433(1), 22-27 (2008).

46 Zeng LH, Xu L, Gutmann DH, Wong M. Rapamycin prevents epilepsy in a mouse model of tuberous sclerosis complex. Ann. Neurol. 63(4), 444-453 (2008).

47 Del Gaudio D, Fang P, Scaglia F et al. Increased mecp2 gene copy number as the result of genomic duplication in neurodevelopmentally delayed males. Genet. Med. 8(12), 784-792 (2006).

48 Friez MJ, Jones JR, Clarkson K et al. Recurrent infections, hypotonia, and mental retardation caused by duplication of mecp2 and adjacent region in xq28. Pediatrics 118(6), e1687-e1695 (2006).

- $\quad$ Effect of Mecp2 duplication in mice.

$49 \mathrm{Na}$ ES, Nelson ED, Adachi M et al. A mouse model for mecp2 duplication syndrome: Mecp2 overexpression impairs learning and memory and synaptic transmission. $J$. Neurosci. 32(9), 3109-3117 (2012).

50 Collins AL, Levenson JM, Vilaythong AP et al. Mild overexpression of mecp2 causes a progressive neurological disorder in mice. Hum. Mol. Genet. 13(21), 2679-2689 (2004).

51 Lioy DT, Garg SK, Monaghan CE et al. A role for glia in the progression of rett/'s syndrome. Nature 475, 497-500 (2011).

52 Kishi N, Macklis JD. Mecp2 is progressively expressed in post-migratory neurons and is involved in neuronal maturation rather than cell fate decisions. Mol. Cell. Neurosci. 27(3), 306-321 (2004).

53 Kay MA, Glorioso JC, Naldini L. Viral vectors for gene therapy: the art of turning infectious agents into vehicles of therapeutics. Nat. Med. 7(1), 33-40 (2001).

54 Kootstra NA, Verma IM. Gene therapy with viral vectors. Annu. Rev. Pharmacol. Toxicol. 43, 413-439 (2003).

55 Blomer U, Naldini L, Verma IM, Trono D, Gage FH. Applications of gene therapy to the cns. Hum. Mol. Genet. 5, 1397-1404 (1996).

56 Mcintyre C, Derrick Roberts AL, Ranieri E, Clements PR, Byers S, Anson DS. Lentiviralmediated gene therapy for murine mucopolysaccharidosis type iiia. Mol. Genet. Metab. 93(4), 411-418 (2008).

57 Brooks AI, Stein CS, Hughes SM et al. Functional correction of established central nervous system deficits in an animal model of lysosomal storage disease with feline immunodeficiency virus-based vectors. Proc. Natl Acad. Sci. USA 99(9), 6216-6221 (2002).

58 Nightingale SJ, Hollis RP, Pepper KA et al. Transient gene expression by nonintegrating lentiviral vectors. Mol. Ther. 13(6), 1121-1132 (2006).

59 Rastegar M, Hotta A, Pasceri P et al. Mecp2 isoform-specific vectors with regulated expression for rett syndrome gene therapy. PLoS ONE 4(8), e6810 (2009).

60 Kantor B, Mccown T, Leone P, Gray SJ. Clinical applications involving cns gene transfer. Adv. Genet. 87, 71-124 (2014).

61 Julu PO, Kerr AM, Apartopoulos F et al. Characterisation of breathing and associated central autonomic dysfunction in the Rett disorder. Arch. Dis. Child. 85(1), 29-37 (2001).

62 Paterson DS, Thompson EG, Belliveau RA et al. Serotonin transporter abnormality in the dorsal motor nucleus of the vagus in Rett syndrome: potential implications for clinical autonomic dysfunction. J. Neuropathol. Exp. Neurol. 64(11), 1018-1027 (2005).

63 Grieger JC, Samulski RJ. Adeno-associated virus as a gene therapy vector: vector development, production and clinical applications. Adv. Biochem. Eng. Biotechnol. 99, 119-145 (2005).

64 Wagner JA, Reynolds T, Moran ML et al. Efficient and persistent gene transfer of aav-cftr in maxillary sinus. Lancet 351(9117), 1702-1703 (1998).

65 Manno CS, Chew AJ, Hutchison S et al. Aav-mediated factor ix gene transfer to skeletal muscle in patients with severe hemophilia b. Blood 101(8), 2963-2972 (2003).

66 Flotte TR, Brantly ML, Spencer LT et al. Phase i trial of intramuscular injection of a recombinant adeno-associated virus alpha 1-antitrypsin (raav2-cb-haat) gene vector to aat-deficient adults. Hum. Gene Ther. 15(1), 93-128 (2004).

67 Sondhi D, Peterson DA, Edelstein AM, Del Fierro K, Hackett NR, Crystal RG. Survival advantage of neonatal cns gene transfer for late infantile neuronal ceroid lipofuscinosis. Exp. Neurol. 213(1), 18-27 (2008).

68 Feigin A, Kaplitt MG, Tang C et al. Modulation of metabolic brain networks after subthalamic gene therapy for parkinson's disease. Proc. Natl Acad. Sci. USA 104(49), 19559-19564 (2007).

69 Muzyczka N, Samulski RJ. Aav-mediated gene therapy for research and therapeutic purposes. Ann. Rev. Virol. 1, 427-451 (2014).

70 Brantly ML, Chulay JD, Wang L et al. Sustained transgene expression despite $\mathrm{t}$ lymphocyte responses in a clinical trial of raavl-aat gene therapy. Proc. Natl Acad. Sci. USA 106(38), 16363-16368 (2009).

71 Nathwani AC, Tuddenham EG, Rangarajan S et al. Adenovirus-associated virus vectormediated gene transfer in hemophilia b. $N$. Engl. J. Med. 365(25), 2357-2365 (2011).

72 Christine CW, Starr PA, Larson PS et al. Safety and tolerability of putaminal aadc gene therapy for parkinson disease. Neurology 73(20), 1662-1669 (2009).

73 Foust KD, Nurre E, Montgomery CL, Hernandez A, Chan CM, Kaspar BK. Intravascular aav9 preferentially targets neonatal neurons and adult astrocytes. Nat. Biotechnol. 27(1), 59-65 (2009).

74 Herzog RW, Hagstrom JN, Kung SH et al. Stable gene transfer and expression of human blood coagulation factor ix after intramuscular injection of recombinant adeno-associated virus. Proc. Natl Acad. Sci. USA 94(11), 5804-5809 (1997).

75 Arruda VR, Stedman HH, Nichols TC et al. Regional intravascular delivery of aav-2-f.Ix to skeletal muscle achieves long-term correction of hemophilia $b$ in a large animal model. Blood 105(9), 3458-3464 (2005).

76 Jiang H, Lillicrap D, Patarroyo-White $\mathrm{S}$ et al. Multiyear therapeutic benefit of aav serotypes 2,6 , and 8 delivering factor viii to hemophilia a mice and dogs. Blood 108(1), 107-115 (2006).

77 Samaco RC, Fryer JD, Ren J et al. A partial loss of function allele of methyl-cpg-binding protein 2 predicts a human 
neurodevelopmental syndrome. Hum. Mol. Genet. 17(12), 1718-1727 (2008).

78 Lawson-Yuen A, Liu D, Han L et al. Ube3a $\mathrm{m} R N A$ and protein expression are not decreased in mecp2r168x mutant mice. Brain Res. 1180, 1-6 (2007).

79 Gray SJ, Foti SB, Schwartz JW et al. Optimizing promoters for recombinant adeno-associated virus-mediated gene expression in the peripheral and central nervous system using self-complementary vectors. Hum. Gene Ther. 22(9), 1143-1153 (2011).

80 Mccarty DM. Self-complementary aav vectors; advances and applications. Mol. Ther. 16(10), 1648-1656 (2008).

81 Adachi M, Keefer EW, Jones FS. A segment of the mecp2 promoter is sufficient to drive expression in neurons. Hum. Mol. Genet. 14(23), 3709-3722 (2005).

82 Liu J, Francke U. Identification of cisregulatory elements for mecp2 expression. Hum. Mol. Genet. 15(11), 1769-1782 (2006).

83 Vitezic M, Bertin N, Andersson R et al. Cage-defined promoter regions of the genes implicated in rett syndrome. BMC Genomics 15(1), 1177 (2014).

84 Andersson R, Gebhard C, Miguel-Escalada I et al. An atlas of active enhancers across human cell types and tissues. Nature 507(7493), 455-461 (2014).

85 Palmer A, Qayumi J, Ronnett G. Mecp2 mutation causes distinguishable phases of acute and chronic defects in synaptogenesis and maintenance, respectively. Mol. Cell. Neurosci. 37(4), 794-807 (2008).

86 Degano AL, Pasterkamp RJ, Ronnett GV. Mecp2 deficiency disrupts axonal guidance, fasciculation, and targeting by altering semaphorin $3 \mathrm{f}$ function. Mol. Cell. Neurosci. 42(3), 243-254 (2009).

87 Baj G, Patrizio A, Montalbano A, Sciancalepore M, Tongiorgi E.

Developmental and maintenance defects in rett syndrome neurons identified by a new mouse staging system in vitro. Front. Cell. Neurosci. 8, 18 (2014).

88 Giacometti E, Luikenhuis S, Beard C, Jaenisch R. Partial rescue of mecp2 deficiency by postnatal activation of mecp2. Proc. Natl Acad. Sci. USA 104(6), 1931-1936 (2007).

89 Lang M, Wither RG, Colic S et al. Rescue of behavioral and eeg deficits in male and female mecp2-deficient mice by delayed mecp2 gene reactivation. Hum. Mol. Genet. 23(2), 303-318 (2014).

- $\mathrm{MeCP} 2$ expression is mandatory to maintain normal neuronal survival.
90 Mcgraw CM, Samaco RC, Zoghbi HY. Adult neural function requires mecp2. Science 333(6039), 186 (2011).

91 Rivera VM, Gao GP, Grant RL et al. Long-term pharmacologically regulated expression of erythropoietin in primates following aav-mediated gene transfer. Blood 105(4), 1424-1430 (2005).

92 Niemeyer GP, Herzog RW, Mount J et al. Long-term correction of inhibitor-prone hemophilia b dogs treated with liver-directed aav2-mediated factor ix gene therapy. Blood 113(4), 797-806 (2009).

93 Stieger K, Schroeder J, Provost N et al. Detection of intact raav particles up to 6 years after successful gene transfer in the retina of dogs and primates. Mol. Ther. 17(3), 516-523 (2009).

94 Leone P, Shera D, Mcphee SW et al. Long-term follow-up after gene therapy for canavan disease. Sci. Transl. Med. 4(165), 165ra163 (2012).

95 Chao HT, Chen H, Samaco RC et al. Dysfunction in gaba signalling mediates autism-like stereotypies and rett syndrome phenotypes. Nature 468(7321), 263-269 (2010).

96 Samaco RC, Mandel-Brehm C, Chao HT et al. Loss of mecp2 in aminergic neurons causes cell-autonomous defects in neurotransmitter synthesis and specific behavioral abnormalities. Proc. Natl Acad. Sci. USA 106(51), 21966-21971 (2009).

97 De Felice C, Rossi M, Leoncini S et al. Inflammatory lung disease in rett syndrome. Mediat. Inflam. 2014, 560120 (2014).

98 Kamal B, Russell D, Payne A et al. Biomechanical properties of bone in a mouse model of rett syndrome. Bone 71, 106-114 (2015).

99 Ramirez JM, Ward CS, Neul JL. Breathing challenges in rett syndrome: lessons learned from humans and animal models. Respir. Physiol. Neurobiol. 189(2), 280-287 (2013).

100 Jefferson A, Fyfe S, Downs J, Woodhead H, Jacoby P, Leonard H. Longitudinal bone mineral content and density in Rett syndrome and their contributing factors. Bone 74 , 191-198 (2015).

101 Justice MJ, Buchovecky CM, Kyle SM, Djukic A. A role for metabolism in rett syndrome pathogenesis: new clinical findings and potential treatment targets. Rare Dis. 1, e27265 (2013).

102 Zhang H, Yang B, Mu X et al. Several raav vectors efficiently cross the blood-brain barrier and transduce neurons and astrocytes in the neonatal mouse central nervous system.
Mol. Ther. 19(8), 1440-1448 (2011).

103 Yang B, Li S, Wang H et al. Global cns transduction of adult mice by intravenously delivered raavrh. 8 and raavrh. 10 and nonhuman primates by raavrh.10. Mol. Ther. 22(7), 1299-1309 (2014).

104 Skene PJ, Illingworth RS, Webb S et al. Neuronal mecp2 is expressed at near histone-octamer levels and globally alters the chromatin state. Mol. Cell 37(4), 457-468 (2010).

105 Pulicherla N, Shen S, Yadav S et al. Engineering liver-detargeted aav9 vectors for cardiac and musculoskeletal gene transfer. Mol. Ther. 19(6), 1070-1078 (2011).

106 Powell SK, Rivera-Soto R, Gray SJ. Viral expression cassette elements to enhance transgene target specificity and expression in gene therapy. Discov. Med. 19(102), 49-57 (2015).

107 Lasalle JM, Goldstine J, Balmer D, Greco CM. Quantitative localization of heterogeneous methyl-cpg-binding protein 2 (mecp2) expression phenotypes in normal and rett syndrome brain by laser scanning cytometry. Hum. Mol. Genet. 10(17), 1729-1740 (2001).

108 Zhou Z, Hong EJ, Cohen S et al. Brainspecific phosphorylation of mecp2 regulates activity-dependent bdnf transcription, dendritic growth, and spine maturation. Neuron 52(2), 255-269 (2006).

109 Ballas N, Lioy DT, Grunseich C, Mandel G. Non-cell autonomous influence of mecp2deficient glia on neuronal dendritic morphology. Nat. Neurosci. 12(3), 311-317 (2009).

110 Maezawa I, Swanberg S, Harvey D, Lasalle JM, Jin LW. Rett syndrome astrocytes are abnormal and spread mecp2 deficiency through gap junctions. J. Neurosci. 29(16), 5051-5061 (2009).

111 Lioy DT, Garg SK, Monaghan CE et al. A role for glia in the progression of Rett's syndrome. Nature 475(7357), 497-500 (2011).

112 Lang M, Wither RG, Brotchie JM, Wu C, Zhang L, Eubanks JH. Selective preservation of mecp2 in catecholaminergic cells is sufficient to improve the behavioral phenotype of male and female mecp2deficient mice. Hum. Mol. Genet. 22(2), 358-371 (2013).

113 Ward CS, Arvide EM, Huang TW, Yoo J, Noebels JL, Neul JL. Mecp2 is critical within hoxb1-derived tissues of mice for normal lifespan. J. Neurosci. 31(28), 10359-10370 (2011). 
114 Goffin D, Brodkin ES, Blendy JA, Siegel SJ, Zhou Z. Cellular origins of auditory event-related potential deficits in rett syndrome. Nat. Neurosci. 17(6), 804-806 (2014).

115 Meins M, Lehmann J, Gerresheim F et al. Submicroscopic duplication in xq28 causes increased expression of the mecp2 gene in a boy with severe mental retardation and features of rett syndrome. J. Med. Genet. 42(2), e12 (2005).

116 Van Esch H, Bauters M, Ignatius J et al. Duplication of the mecp2 region is a frequent cause of severe mental retardation and progressive neurological symptoms in males. Am. J. Hum. Genet. 77(3), 442-453 (2005).

117 Philippe C, Villard L, De Roux N et al. Spectrum and distribution of mecp2 mutations in 424 rett syndrome patients: a molecular update. Eur. J. Med. Genet. 49(1), 9-18 (2006).

118 Martin R, Mogg AE, Heywood LA, Nitschke L, Burke JF. Aminoglycoside suppression at uag, uaa and uga codons in escherichia coli and human tissue culture cells. Mol. Gen. Genet. 217(2-3), 411-418 (1989).

119 Manuvakhova M, Keeling K, Bedwell DM. Aminoglycoside antibiotics mediate context-dependent suppression of termination codons in a mammalian translation system. RNA 6(7), 1044-1055 (2000).

120 Brendel C, Klahold E, Gartner J, Huppke P. Suppression of nonsense mutations in rett syndrome by aminoglycoside antibiotics. Pediatr. Res. 65(5), 520-523 (2009).

121 Popescu AC, Sidorova E, Zhang G, Eubanks JH. Aminoglycoside-mediated partial suppression of mecp2 nonsense mutations responsible for rett syndrome in vitro. J. Neurosci. Res. 88(11), 2316-2324 (2010).

122 Welch EM, Barton ER, Zhuo J et al. Ptc124 targets genetic disorders caused by nonsense mutations. Nature 447(7140), 87-91 (2007).

123 Nudelman I, Rebibo-Sabbah A, Cherniavsky $\mathrm{M}$ et al. Development of novel aminoglycoside (nb54) with reduced toxicity and enhanced suppression of disease-causing premature stop mutations. J. Med. Chem. 52(9), 2836-2845 (2009).
124 Brendel C, Belakhov V, Werner $\mathrm{H}$ et al. Readthrough of nonsense mutations in rett syndrome: evaluation of novel aminoglycosides and generation of a new mouse model. J. Mol. Med. 89(4), 389-398 (2011).

125 Mohandas T, Sparkes RS, Shapiro LJ. Reactivation of an inactive human $\mathrm{x}$ chromosome: evidence for $\mathrm{x}$ inactivation by DNA methylation. Science 211 (4480), 393-396 (1981).

126 Bhatnagar S, Zhu X, Ou J et al. Genetic and pharmacological reactivation of the mammalian inactive $\mathrm{x}$ chromosome. Proc. Natl Acad. Sci. USA 111(35), 12591-12598 (2014).

127 Sakuma T, Nishikawa A, Kume S, Chayama $\mathrm{K}$, Yamamoto T. Multiplex genome engineering in human cells using all-in-one crispr/cas9 vector system. Sci. Rep. 4, 5400 (2014).

128 Mali P, Yang L, Esvelt KM et al. Rna-guided human genome engineering via cas 9 . Science 339 (6121), 823-826 (2013).

129 Sorek R, Kunin V, Hugenholtz P. Crispr - a widespread system that provides acquired resistance against phages in bacteria and archaea. Nat. Rev. Microbiol. 6(3), 181-186 (2008).

130 Horvath P, Barrangou R. Crispr/cas, the immune system of bacteria and archaea. Science 327(5962), 167-170 (2010).

131 Wang H, Yang H, Shivalila CS et al. One-step generation of mice carrying mutations in multiple genes by crispr/cas-mediated genome engineering. Cell 153(4), 910-918 (2013).

132 Swiech L, Heidenreich M, Banerjee A et al. In vivo interrogation of gene function in the mammalian brain using crispr-cas9. Nat. Biotechnol. 33(1), 102-106 (2015).

133 Schwank G, Koo BK, Sasselli V et al. Functional repair of cftr by crispr/cas9 in intestinal stem cell organoids of cystic fibrosis patients. Cell Stem Cell 13(6), 653-658 (2013).

134 Yin $\mathrm{H}$, Xue W, Chen $\mathrm{S}$ et al. Genome editing with cas 9 in adult mice corrects a disease mutation and phenotype. Nat. Biotechnol. 32(6), 551-553 (2014).
135 San Filippo J, Sung P, Klein H. Mechanism of eukaryotic homologous recombination. Annu. Rev. Biochem. 77, 229-257 (2008).

136 Jeppesen DK, Bohr VA, Stevnsner T. DNA repair deficiency in neurodegeneration. Prog. Neurobiol. 94(2), 166-200 (2011).

137 Fu Y, Foden JA, Khayter C et al. Highfrequency off-target mutagenesis induced by crispr-cas nucleases in human cells. Nat. Biotechnol. 31(9), 822-826 (2013).

138 Cho SW, Kim S, Kim Y et al. Analysis of off-target effects of crispr/cas-derived rna-guided endonucleases and nickases. 24(1), 132-141 (2014).

139 Derecki NC, Cronk JC, Lu Z et al. Wild-type microglia arrest pathology in a mouse model of rett syndrome. Nature 484, 105-109 (2012).

140 Wang J, Wegener JE, Huang TW et al. Wild-type microglia do not reverse pathology in mouse models of rett syndrome. Nature 521(7552), E1-E4 (2015).

141 Yu C, Liu Y, Ma T et al. Small molecules enhance crispr genome editing in pluripotent stem cells. Cell Stem Cell 16(2), 142-147 (2015).

142 Maresca M, Lin VG, Guo N, Yang Y. Obligate ligation-gated recombination (obligare): custom-designed nucleasemediated targeted integration through nonhomologous end joining. Genome Res. 23(3), 539-546 (2013).

143 Tiyaboonchai A, Mac H, Shamsedeen R et al. Utilization of the aavs1 safe harbor locus for hematopoietic specific transgene expression and gene knockdown in human es cells. Stem Cell Res. 12(3), 630-637 (2014).

144 Makarova KS, Haft DH, Barrangou R et al. Evolution and classification of the crispr-cas systems. Nat. Rev. Microbiol. 9(6), 467-477 (2011).

145 Ran FA, Cong L, Yan WX et al. In vivo genome editing using staphylococcus aureus cas9. Nature 520(7546), 186-191 (2015). 
